Vol. 30 No. 2 September, 1985

\title{
Japanese Journal
}

\section{of \\ Physical Education}

\section{Contents}

Review

105 Akira Nakagawa

Present status and perspective of study on decision making in ball games

Originals

117 Yuji Yamamoto, Shiro Nakagomi, Takashi Ino and Toshimi Kudo

A re-examination of inverted. $U$ hypothesis by attentional narrowing phenomenon

129 Akiliko Ishihara. Shigeru Katsuta and Tatsumori Fujita

Effects of intermittent-running training on the histochemical properties of nerve cell and muscle fibre

137 Sajoko Hayamizu, Chikashi Yamamoto. Tetsuo Ohkuwa, Koichi Katsumata and Yuzo Sato Biochemical estimation of training effects

145 Masahiro Doi, Kojiro Koga, Noriyuki Monma, Masayo Asano and Ichiro Watanabe

Materials A study on mechanical and stroke characteristics of the tennis racket (2nd report)

153 Tojohiko Ito

Factorical structure of attributional styles in sport situations and its characteristics

161 Takahiko Nishijima, Yoshiyuki Matsuura and Seiji Ohsawa

The determining factors of team performance and their relationship with game in vol. leyball

News and Informations of J.S. P. E.

Edited by

Japanese Society of Physical Education

Tokyo, Japan 